TRANSACTIONS OF THE

AMERICAN MATHEMATICAL SOCIETY

Volume 358, Number 7, Pages 2917-2926

S 0002-9947(06)04195-X

Article electronically published on February 14, 2006

\title{
ON HÖLDER CONTINUOUS RIEMANNIAN AND FINSLER METRICS
}

\author{
ALEXANDER LYTCHAK AND ASLI YAMAN
}

\begin{abstract}
We discuss smoothness of geodesics in Riemannian and Finsler metrics.
\end{abstract}

\section{INTRODUCTION}

In this paper we improve and generalize a result of Calabi and Hartmann in CH70. They study smoothness of isometries between equal-dimensional manifolds with $\alpha$-Hölder continuous Riemannian metrics and prove that each isometry is of class $\mathcal{C}^{1, \alpha}$. The main tool in the proof is Theorem 3.1 of [CH70, stating that geodesics in $\alpha$-Hölder Riemannian metrics are uniformly $\mathcal{C}^{1, \alpha}$, but the proof of this theorem is not correct. Although the result on the smoothness of isometries is true, as Taylor has shown by a different method in Tay, Theorem 3.1 is wrong for $\alpha<1$.

Indeed one of our results is the following:

Theorem 1.1. For each $0<\alpha \leq 1$ set $\beta=\frac{\alpha}{2-\alpha}$. There is an $\alpha$-Hölder Riemannian metric on $\mathbb{R}^{2}$, such that geodesics near the origin are not uniformly $\mathcal{C}^{1, l}$ for any $l>\beta$.

Actually it seems to be possible but technically not trivial to construct an $\alpha$ Hölder continuous Riemannian metric for which some geodesic $\gamma$ is not $\mathcal{C}^{1, l}$ for all $l>\beta$. This would show that there are manifolds (of different dimensions) with $\alpha$-Hölder Riemannian metrics and a distance-preserving embedding of one of them into another that is at best $\mathcal{C}^{1, \beta}$, in contrast to the $\mathcal{C}^{1, \alpha}$ smoothness of isometries shown by Taylor.

Remark 1.1. If $\alpha$ goes to 1 , then the regularity of geodesics is almost $\mathcal{C}^{1, \alpha}$, whereas if $\alpha$ goes to 0 , one only gets a bit more than half of the expected regularity!

In contrast to this result the first author proved in Lyt that geodesics in $\mathcal{C}^{1, \alpha}$ submanifolds of smooth Riemannian manifolds are uniformly $\mathcal{C}^{1, \alpha}$. This gives us the following non-embeddability result:

Corollary 1.2. For $0<\alpha<1$ and $\beta=\frac{\alpha}{2-\alpha}$ there are $\alpha$-Hölder continuous Riemannian metrics that (even locally) do not admit $\mathcal{C}^{1, l}$ arcwise isometric embeddings into any smooth Riemannian manifold, for any $l>\beta$.

Received by the editors March 25, 2004.

2000 Mathematics Subject Classification. Primary 53B40, 53B20.

Key words and phrases. Geodesics, isometric embeddings.

(C)2006 American Mathematical Society Reverts to public domain 28 years from publication 
This result should be compared to the theorems of Nash, stating that each manifold with a continuous Riemannian metric has a $\mathcal{C}^{1}$ arcwise isometric embedding into some $\mathbb{R}^{n}$ and that for $k \geq 3$ each Riemannian manifold with a $\mathcal{C}^{k}$-Riemannian metric has a $\mathcal{C}^{k}$ arcwise isometric embedding into some Euclidean space.

Remark 1.2. For $\alpha=1$ a statement similiar to Corollary 1.2 still holds, but for another reason. Namely, it is shown in Lyt that each $\mathcal{C}^{1,1}$ submanifold of a smooth Riemannian manifold has curvature bounded from both sides with respect to the inner metric, whereas it is certainly not true even for a general Riemannian metric of class $\mathcal{C}^{1, l}$, for any fixed $l<1$.

The proof of Theorem 3.1 in CH70 only shows that geodesics in $\alpha$-Hölder Riemannian metrics are uniformly $\mathcal{C}^{1, \frac{\alpha}{2}}$. We repeat their argument and generalize this result to sufficiently convex Finsler metrics (see Definition 2.3 and Definition 3.1 for precise definitions):

Theorem 1.3. Let $(M,|\cdot|)$ be a manifold with an $\alpha$-Hölder Finsler structure $|\cdot|$, such that the norms $|\cdot|_{x}$ are locally uniformly of convexity type $p$. Then geodesics with respect to the Finsler metric in $M$ are locally uniformly $\mathcal{C}^{1, \frac{\alpha}{p}}$.

Exactly as in the last part of [CH70, Theorem 1.3 implies that every distancepreserving embedding between two manifolds as in Theorem 1.3 is $\mathcal{C}^{1, \frac{\alpha}{p}}$.

Finally we prove the main result of this paper, showing that Theorem 1.1 is optimal:

Theorem 1.4. Let $\left(M,\langle\cdot, \cdot\rangle_{x}\right)$ be a manifold with an $\alpha$-Hölder Riemannian metric. Then geodesics in $M$ are locally uniformly $\mathcal{C}^{1, \beta}$ for $\beta=\frac{\alpha}{2-\alpha}$.

After recalling some basic facts about $\mathcal{C}^{1, \alpha}$ maps, we study curves in a manifold $M$ with a Finsler structure and compare the lengths of curves to their lengths measured in some tangent space. Using these estimations we prove Theorem 1.3 and Theorem 1.4. The main idea is that a curve $\gamma:[a, b] \rightarrow M$ that is not $\mathcal{C}^{1, l}$ admits for arbitrary $A>0$ arbitrary closed points $t, s \in[a, b]$ such that $\gamma\left(\frac{t+s}{2}\right)$ is at least $A\|t-s\|^{l}$ far away from the straight line $\eta$ connecting $\gamma(t)$ and $\gamma(s)$. Considering a Banach space metric on $M$ induced by a norm $\mid \cdot{ }_{x}$ for each $x$, we see from the fact that the norms are sufficiently convex, that the length of $\gamma$ is much bigger than the length of $\eta$ with respect to this norm. Now using the fact that the lengths of $\eta$ and $\gamma$ with respect to this norm do not differ too much from their actual lengths in $M$, one gets a contradiction if $\gamma$ is a geodesic. The idea of Hartman and Calabi, in order to prove Theorem 1.3. was to compute the lengths of $\eta$ and $\gamma$ relative to a tangent space at the fixed point $\gamma\left(t_{0}\right)$. A better estimation providing Theorem 1.4 is achieved by comparing at each $t$ the quantities $\left|\gamma^{\prime}(t)\right|_{\gamma(t)}$ and $\left|\eta^{\prime}(t)\right|_{\eta(t)}$.

After preliminaries in Section 2, in Section 3 we study Hölder continuous Finsler structures, discuss examples and basics of the structure and recall the comparisons of Calabi and Hartmann of lengths of curves measured in $M$, with their lengths with respect to the constant Finsler structure $|\cdot|_{x}=|\cdot|_{x_{0}}$. In Section 4 we prove Theorem 1.3 and Theorem 1.4. Finally in Section 5 we prove Theorem 1.1 by giving a counterexample to the result of Calabi and Hartman.

We are grateful to Werner Ballmann and Juan Souto for helpful suggestions. We are indebted to Reiner Schätzle for indicating a mistake in the previous version of 
our counterexample. We are also thankful to Jonathan Wahl for bringing the work of Michael Taylor to our attention.

\section{Preliminaries}

Let us start with some basic conventions that shall be followed throughout this work. A geodesic in a metric space $X$ is an arclength parametrised curve $\gamma:[a, b] \rightarrow$ $X$ satisfying $d(\gamma(a), \gamma(b))=b-a$. By $\|\cdot\|$ we will denote the Euclidean norm in Euclidean spaces. Given a $\mathcal{C}^{t}$ manifold $M$ a chart is a $\mathcal{C}^{t}$ diffeomorphism which sends an open subset of $\mathbb{R}^{n}$ onto an open subset of $M$. We shall always omit the neighbourhood in $M$ and identify a chart with its image in $\mathbb{R}^{n}$.

2.1. Hölder maps. A map $f:\left(M, d_{1}\right) \rightarrow\left(N, d_{2}\right)$ between metric spaces $M$ and $N$ is $(C, \alpha)$-Hölder, for $C>0,1 \geq \alpha>0$, if for all $x, z \in X$ one has $d_{2}(f(x), f(z)) \leq$ $C d_{1}(x-z)^{\alpha}$. The map is called locally $\alpha$-Hölder if $M$ is covered by open subsets $U_{j}$, such that each restriction $f: U_{j} \rightarrow N$ is $\left(C_{j}, \alpha\right)$-Hölder for some $C_{j}=C\left(U_{j}\right)$.

Let $M$ and $N$ be locally compact spaces. Let $\mathcal{F}$ be a family of maps $f_{a}: D_{a} \rightarrow N$ defined on subsets $D_{a}$ of $M$. For subsets $X \subset M$ and $Y \subset N$ denote by $\mathcal{F}_{X, Y}$ the subfamily of all maps $f \in \mathcal{F}$ satisfying $f_{a}\left(X \cap D_{a}\right) \subset Y$.

Definition 2.1. We will say that the family $\mathcal{F}$ is locally uniformly $\alpha$-Hölder, if for all compact subsets $X \subset M$ and $Y \subset N$ there is a positive $C=C(X, Y)>0$, such that the restriction $f_{a}:\left(D_{a} \cap X\right) \rightarrow Y$ is $(C, \alpha)$-Hölder for all $f_{a} \in \mathcal{F}_{X, Y}$.

Since $M$ and $N$ are by definition locally compact, the family $\mathcal{F}$ is locally uniformly $\alpha$-Hölder if and only if the condition of Definition 2.1 is satisfied for all open relatively compact subsets $X=U_{j}$ and $Y=V_{i}$ of a covering $U_{j}$ of $M$, resp. $V_{i}$ of $N$.

2.2. $\mathcal{C}^{1, \alpha}$-maps. A map $f: U \rightarrow \mathbb{R}^{m}$ defined on an open subset $U$ of $\mathbb{R}^{n}$ is locally $\mathcal{C}^{1, \alpha}$ if it is $\mathcal{C}^{1}$ and its differential $D f: U \rightarrow \mathbb{L}\left(\mathbb{R}^{n}, \mathbb{R}^{m}\right)$ is locally $\alpha$-Hölder where $1 \geq \alpha>0$.

The following lemma is in fact a consequence of a more general Lemma 2.2 . However we state the lemma and give a proof for later reference in the case of curves.

Lemma 2.1. Let $\gamma:[a, b] \rightarrow \mathbb{R}^{n}$ be a $\mathcal{C}^{1}$ curve with $\left\|\gamma^{\prime}(t)-\gamma^{\prime}(s)\right\| \leq C\|s-t\|^{\alpha}$ for all $s, t \in(a, b)$. Then for all $r$ with $a<r<b$ we have $\left\|\gamma^{\prime}(r)\right\| b-a\|-(\gamma(b)-\gamma(a))\| \leq$ $C\|b-a\|^{1+\alpha}$.

Proof. We have $\gamma(b)-\gamma(a)=\int_{a}^{b} \gamma^{\prime}(x) d x=\int_{a}^{b} \gamma^{\prime}(r) d x+\int_{a}^{b}\left(\gamma^{\prime}(x)-\gamma^{\prime}(r)\right) d x$. Using $\left\|\gamma^{\prime}(x)-\gamma^{\prime}(r)\right\| \leq C\|b-a\|^{\alpha}$ we obtain the result.

In particular this shows that under the conditions of Lemma 2.1 the distance between $\gamma(r)$ and the affine line through $\gamma(a)$ and $\gamma(b)$ is at most $C|b-a|^{1+\alpha}$.

Definition 2.2. A family $\mathcal{F}$ of maps $f_{a}: U_{a} \rightarrow \mathbb{R}^{m}$ defined on open subsets $U_{a}$ of $\mathbb{R}^{n}$ is called locally uniformly $\mathcal{C}^{1, \alpha}$ if the family is locally uniformly Lipschitz and the family $\tilde{\mathcal{F}}$ of differentials $\tilde{f}_{a}=\left(f_{a}, D f_{a}\right): U_{a} \rightarrow \mathbb{R}^{m} \times L\left(\mathbb{R}^{n}, \mathbb{R}^{m}\right)$ is locally uniformly $\alpha$-Hölder.

We will need the following characterisation of locally uniformly $\mathcal{C}^{1, \alpha}$ families. That result is given as Lemma 2.1 in CH70. 
Lemma 2.2. A family $\mathcal{F}$ of locally uniformly Lipschitz maps $f_{a}: U_{a} \rightarrow \mathbb{R}^{m}$ defined on open subsets $U_{a}$ of $\mathbb{R}^{n}$ is locally uniformly $\mathcal{C}^{1, \alpha}$, if for each ball $B \subset \mathbb{R}^{m}$, one can cover $U_{a}$ by open subsets $O_{j}$, such that for each $f \in \mathcal{F}_{O_{j}, B}$, all $x \in O_{j}$ and all $h \in R^{n}$ such that $x+h$ and $x-h$ are in $O_{j}$, one has $\|f(x+h)+f(x-h)-2 f(x)\| \leq$ $C\|h\|^{1+\alpha}$ for some constant $C=C\left(O_{j}\right)$.

Since the composition of $\mathcal{C}^{1, \alpha}$ maps is again $\mathcal{C}^{1, \alpha}$, the notion of a $\mathcal{C}^{1, \alpha}$ manifold is well defined. Moreover one can speak about locally uniformly $\mathcal{C}^{1, \alpha}$ families of maps between two $\mathcal{C}^{1, \alpha}$ manifolds.

2.3. Norms of convexity type $p$. Let $(V,|\cdot|)$ be a (finite-dimensional) normed vector space. Recall that the modulus of convexity $\delta=\delta_{|\cdot|}$ of the norm is defined by

$$
\delta(\epsilon)=\inf \left\{1-\frac{|v+w|}{2}|| v|,| w|\leq 1,| v-w \mid \geq \epsilon\right\} .
$$

(See [LT79, pp. 59 - 66, for further discussion on this.)

Definition 2.3. Let $\kappa>0, p \geq 2$ be given. We will say that the norm $|\cdot|$ is $\kappa$-convex of type $p$, if $\delta(\epsilon) \geq \kappa \epsilon^{p}$ for all $\epsilon<\kappa$.

Remark 2.1. Informally speaking, the above definition means that $V$ is at least as convex as the Lebesgue space $\mathcal{L}^{p}$ (see [LT79] p. 63).

Example 2.2. If $(V,|\cdot|)$ is a $\kappa$-convex space of type $p$, then the same holds for each subspace of $V$. On the other hand $(V,|\cdot|)$ is $\kappa$-convex of type $p$ if this is true for each two-dimensional subspace $W$ of $V$.

Example 2.3. The Euclidean space is $\kappa$-convex of type 2 with $\kappa=\frac{1}{10}$. More generally, the statement that the norm on $V$ is $\kappa$-convex of type 2 is equivalent to the fact that the second fundamental form of the unit sphere $S$ of $V$ is bounded below by a constant $\epsilon=\epsilon(\kappa)>0$.

We can now reformulate the definition in a way we will use it:

Lemma 2.3. Let the norm $|\cdot|$ on the vector space $V$ be $\kappa$-convex of type $p$. Let $K, \alpha>0$. Then there are positive $\epsilon=\epsilon(\kappa, K, \alpha)$ and $\lambda=\lambda(\kappa, K, \alpha)$ such that for all numbers $h$ with $h \leq \epsilon$ and all $v, w \in V$ with $|v|,|w| \leq h+K h^{1+\alpha}$ and $|v+w| \geq 2 h-K h^{1+\alpha}$, one has $|v-w| \leq \lambda h^{1+\frac{\alpha}{p}}$.

Proof. First we note that if $|\cdot|$ is $\kappa$-convex of type $p$, then for all $H \leq \kappa^{p+1}=\epsilon^{\prime}$ and for all $\left|v_{0}\right|,\left|w_{0}\right| \leq 1$ the inequality $2-\left|v_{0}+w_{0}\right| \leq H$ implies $\left|v_{0}-w_{0}\right| \leq \lambda^{\prime} H^{\frac{1}{p}}$ where $\lambda^{\prime}=\kappa^{-\frac{1}{p}}$.

Now consider $v_{0}=\frac{v}{h+K h^{1+\alpha}}$ and $w_{0}=\frac{w}{h+K h^{1+\alpha}}$. Then $\left|v_{0}\right|,\left|w_{0}\right| \leq 1$ and $2-\left|v_{0}+w_{0}\right| \leq 3 K h^{\alpha}$. Thus for $h$ with $3 K h^{\alpha} \leq \epsilon^{\prime}$ the above remark implies that $\left|v_{0}-w_{0}\right| \leq \lambda^{\prime}\left(3 K h^{\alpha}\right)^{\frac{1}{p}}=\left(\frac{3 K}{\kappa}\right)^{\frac{1}{p}} h^{\frac{\alpha}{p}}$. Thus by setting $\epsilon=\left(\frac{\epsilon^{\prime}}{3 K}\right)^{\frac{1}{\alpha}}$ and $\lambda=\left(\frac{3 K}{\kappa}\right)^{\frac{1}{p}}$, we have the result.

\section{Finsler structures}

3.1. Definition and examples. A Finsler structure on a $\mathcal{C}^{1}$-manifold $M$ is a continuous map $|\cdot|: T M \rightarrow[0, \infty)$ on the tangent bundle $T M$ such that for all $x \in M$ the restriction $|\cdot|_{x}: T_{x} M \rightarrow[0, \infty)$ is a norm. Given a Finsler structure one can speak about lengths of locally Lipschitz curves $\gamma:[a, b] \rightarrow M$ given by 
$L(\gamma)=\int_{a}^{b}\left|\gamma^{\prime}(t)\right|_{\gamma(t)} d t$. This defines as usual a length metric $d$ on $M$ which is called the Finsler metric. Remark that the Finsler metrics defined by two different Finsler structures are locally bi-Lipschitz equivalent. In particular the Finsler metric defines the usual locally compact topology on $M$. Thus by the theorem of Arzela-Ascoli $M$ is covered by open sets $U_{j}$, such that each two points $x, z \in U_{j}$ are connected in $M$ by a geodesic.

Definition 3.1. A Finsler structure $|\cdot|$ on a manifold $M$ is locally uniformly of type $p$, if for each compact subset $X$ of $M$ there is a constant $\kappa=\kappa(X)>0$ such that, for each $x \in X$, the norm $|\cdot|_{x}$ is $\kappa$-convex of type $p$.

For an immersion $\phi: N \rightarrow M$ the Finsler structure on $M$ defines a Finsler structure on $N$ through $|v|_{x}=\left|D_{x} \phi(v)\right|_{\phi(x)}$. In particular we get a Finsler structure on each $\mathcal{C}^{1}$-submanifold of $M$ and on each chart $U \subset \mathbb{R}^{n}$ of $M$.

On an open subset $U$ of $\mathbb{R}^{n}$ with a Finsler structure $|\cdot|$ we consider the function $\tilde{o}$ : $U \times U \rightarrow \mathbb{R}^{+}$given by $\tilde{o}(x, y)=\sup _{\|v\| \leq 1}|||v|_{x}-|v|_{y}||$. Recall that $\|\cdot\|$ denotes the Euclidean norm. The function $\tilde{o}$ is continuous and vanishes on the diagonal. Hence on each relatively compact open subset $V$ of $U$ the function $o_{V}(r)=\sup \{\tilde{o}(x, y) \mid$ $x, y \in V,\|x-y\| \leq r\}$ is finite, continuous, non-decreasing and satisfies $o_{V}(0)=0$. Moreover, since $V$ is relatively compact, we can find a constant $C>0$ such that $\frac{1}{C}|v|_{x} \leq\|v\| \leq C|v|_{x}$ for all $v \in \mathbb{R}^{n}$ and all $x \in V$.

Let $W$ be an open subset of $\mathbb{R}^{m}$ and let $\phi: W \rightarrow V$ be an immersion, satisfying $\|\phi(x)-\phi(y)\| \leq L\|x-y\|$ for some constant $L \leq 0$. Consider the quantity $u(r)=$ $\sup _{\|v\| \leq 1,\|x-y\| \leq r}\left\|D_{x} \phi(v)-D_{y} \phi(v)\right\|$. Then for the induced Finsler structure on $W$ it is easily checked that $o_{W}(r) \leq L o_{V}(L r)+C \cdot u(r)$.

Hence if for all $r$ we have $o_{V}(r) \leq C r^{\alpha}$ for some $C \geq 0$ and $1 \geq \alpha>0$, and if the map $\phi$ is $\mathcal{C}^{1, \alpha}$, we obtain $o_{W}(r) \leq C^{\prime} r^{\alpha}$ for some $C^{\prime} \geq 0$. Hence the following definition is meaningful.

Definition 3.2. A Finsler structure on a $\mathcal{C}^{1, \alpha}$-manifold $M$ is called a $\mathcal{C}^{\alpha}$-Finsler structure if for each chart $U$ and for each relatively compact open subset $V$ of $U$ one has $o_{V}(r) \leq C r^{\alpha}$ for all $r \geq 0$ and some constant $C=C(V)$.

In other words, for all $v \in \mathbb{R}^{n}$ with $\|v\| \leq 1$ and for all $x, y$ in a relatively compact set $V$ of $U$, one has $\left\||v|_{x}-|v|_{y}|| \leq C|| x-y\right\|^{\alpha}$ for some constant $C=C(V)$.

From the considerations above the following is clear.

Example 3.1. Let $M$ be a $\mathcal{C}^{1, \alpha}$ manifold with a $\mathcal{C}^{\alpha}$ Finsler structure. If $\phi: N \rightarrow M$ is a $\mathcal{C}^{1, \alpha}$ immersion, then the induced Finsler structure on $N$ is $\mathcal{C}^{\alpha}$. In particular each $\mathcal{C}^{1, \alpha}$ submanifold of $M$ inherits a $\mathcal{C}^{\alpha}$ Finsler structure.

The next example is immediately checked in a fixed chart.

Example 3.2. Let $M$ be a $\mathcal{C}^{1, \alpha}$ manifold with a $\mathcal{C}^{\alpha}$ Finsler structure. Then for a locally $\alpha$-Hölder function $f: M \rightarrow R^{+}$the Finsler structure $|\cdot|^{\prime}:=f|\cdot|$ on $M$ given by $|v|_{x}^{\prime}=f(x)|v|_{x}$ is again $\mathcal{C}^{\alpha}$.

The last example is probably the most interesting one.

Example 3.3. Let $M_{1}, M_{2}$ be two manifolds with boundaries $N_{1}, N_{2}$. Let $g_{1}$ resp. $g_{2}$ be Riemannian metrics on $M_{1}$ and on $M_{2}$. Assume that there is a $\mathcal{C}^{1, \alpha}$ diffeomorphism $\phi: N_{1} \rightarrow N_{2}$ with $\phi^{*}\left(g_{2}\right)=g_{1}$. Consider the manifold $M$ that arises from gluing $M_{1}$ and $M_{2}$ along the identifications of their boundaries given 
by the isometry $\phi$. The manifold $M$ is $\mathcal{C}^{1, \alpha}$, and the Riemannian metric $g$ that coincides with $g_{1}$ on $M_{1}$ and with $g_{2}$ on $M_{2}$ is $\mathcal{C}^{\alpha}$. It is interesting to note that even if $M, N_{1}, N_{2}$ and $\phi$ are smooth, the resulting metric $g$ is not better than Lipschitz. It can be smooth only in very special cases. Usually it does not even have locally one-sided curvature bounds (compare Kos02b and Kos02a]).

3.2. Comparison to the tangent spaces. Since all our results are of local nature we will restrict ourselves for the rest of the section to a chart $U \subset \mathbb{R}^{n}$, that is, a convex subset of $\mathbb{R}^{n}$. So let $|\cdot|$ be a continuous Finsler structure on $U$. We can assume (considering a relatively compact subset of $U$ instead of $U$ ) that the function $o_{U}(r)=o(r)$ as defined above is finite and bounded above by $C_{1}>2$ for all $r$. Moreover, for all $x \in U$ we have $\frac{1}{C_{2}}\|\cdot\| \leq|\cdot|_{x} \leq C_{2}\|\cdot\|$ for some constant $C_{2} \geq 2$. Hence for the Finsler metric $d$ and all $x, y \in U$ one has $\frac{1}{C_{2}} d(x, y) \leq$ $\|x-y\| \leq C_{2} d(x, y)$.

For a Lipschitz curve $\gamma:[a, b] \rightarrow U$ we will denote its Euclidean length by $L_{\epsilon}(\gamma)$. For each $x \in U$ we denote by $L^{x}(\gamma)$ the length of $\gamma$ in the normed space $\left(\mathbb{R}^{n},|\cdot|_{x}\right)$, i.e. $L^{x}(\gamma)=\int_{a}^{b}\left|\gamma^{\prime}(t)\right|_{x} d t$. We will refer to this length as the length relative to $x$. From our assumptions on $U$ we see that $\frac{1}{C_{2}} L_{\epsilon}(\gamma) \leq L(\gamma) \leq C_{2} L_{\epsilon}(\gamma)$.

The following lemma allows us to control the relative lengths of curves in terms of their actual lengths.

Lemma 3.1. Let $\gamma:[0, s] \rightarrow U$ be a Lipschitz curve starting at $x=\gamma(0)$. Then we have $\left\|L(\gamma)-L^{x}(\gamma)\right\| \leq o\left(L_{\epsilon}(\gamma)\right) L_{\epsilon}(\gamma)$.

Proof. We may assume that $\gamma$ is parametrized by the Euclidean length. Then $L(\gamma)=\int_{0}^{s}\left|\gamma^{\prime}(t)\right|_{\gamma(t)} d t=L^{x}(\gamma)+\int_{0}^{s}\left(\left|\gamma^{\prime}(t)\right|_{\gamma(t)}-\left|\gamma^{\prime}(t)\right|_{x}\right) d t$.

However, by definition we get $\left\|\left|\gamma^{\prime}(t)\right|_{\gamma(t)}-\left|\gamma^{\prime}(t)\right|_{x}\right\| \leq o(\| x-\gamma(t)||)$. Since $\|x-\gamma(t)\| \leq t$ and the function $o$ is non-decreasing, we get $\left\|L(\gamma)-L^{x}(\gamma)\right\| \leq$ $\int_{0}^{s} o(t) d t \leq o(s) s=o\left(L_{\epsilon}(\gamma)\right) L_{\epsilon}(\gamma)$.

Using the same argument of the proof of Lemma 3.1 one can also prove the following lemma, which allows us to compare the relative lengths of a curve.

Lemma 3.2. Let $\gamma:[0, s] \rightarrow U$ be a Lipschitz curve. Then

$$
L^{x}(\gamma)-L^{y}(\gamma) \leq o(\|x-y\|) L_{\epsilon}(\gamma)
$$

for all $x, y \in U$.

The following results are immediate applications of Lemma 3.1 .

Corollary 3.3. For all $x, y \in U$ one has $\left\|d(x, y)-|x-y|{ }_{x}\right\| \leq o(\|x-y\|)\|x-y\|$, where d denotes the Finsler metric.

In the case $o(r) \leq C r^{\alpha}$, where $C=C(U)$ is a constant depending only $U$ and $1 \geq \alpha>0$, we obtain:

Corollary 3.4. If the Finsler structure on $U$ is $C^{\alpha}$, then $\left\|L(\gamma)-L^{x}(\gamma)\right\| \leq$ $C^{\prime} L(\gamma)^{1+\alpha}$ and $\left\|d(x, z)-|x-z|_{x}\right\| \leq C^{\prime} d(x, z)^{1+\alpha}$ for $C^{\prime}=C_{2}{ }^{\alpha+1} C$.

\section{The SMOothness of GeOdesics}

Let $M$ be a manifold with a $\mathcal{C}^{\alpha}$ Finsler structure. We have already noted that the statements are of local nature. Therefore from now on we fix a chart $U$ on $M$ and assume that $U$ is an open ball in $\mathbb{R}^{n}$ with a $\mathcal{C}^{\alpha}$ Finsler structure. We denote 
by $d$ the Finsler metric and by $\|\cdot\|$ the Euclidean metric on $U$, and $|\cdot|_{x}$ denotes the norm at point $x$ induced from the Finsler structure. We assume that all the constants introduced in Section 3.2 are the same. Thus let $C>2$ be a constant, such that for all $x \in U$ we have $\frac{1}{C}\|\cdot\| \leq \mid \cdot{ }_{x} \leq C\|\cdot\|$ and that the function $o$ satisfies $o(r)=o_{U}(r) \leq C r^{\alpha}$. We have $\frac{1}{C} d(x, y) \leq\|x-y\| \leq C d(x, y)$ and $\left\|d(x, y)-|x-y|_{x}\right\| \leq\|x-y\|^{1+\alpha}$. Moreover, we assume that for all $x, y$ in $U$ and for all Lipschitz curves $\gamma$ in $U$ starting at $x$ one has $\left\|L^{x}(\gamma)-L(\gamma)\right\| \leq C L^{x}(\gamma)^{1+\alpha}$ and $\left\|L^{x}(\gamma)-L(\gamma)\right\| \leq C L(\gamma)^{1+\alpha}$. Also $\left\|d(x, y)-|x-y|_{x}\right\| \leq C d(x, y)^{1+\alpha}$ and $\||x-y|_{x}-|x-y|_{y}|| \leq C|| x-y||^{1+\alpha}$ for all $x, y \in U$.

4.1. The estimations of Hartmann and Calabi and the proof of Theorem 1.3. Here we assume in addition to the above hypothesis that for each $x \in U$ the norm $|\cdot|_{x}$ is $\kappa$-convex of type $p$. Let $h<1$ be a small positive number and let $\gamma:[-h, h] \rightarrow U$ be a geodesic. Set $x=\gamma(-h), y=\gamma(0)$ and $z=\gamma(h)$. Let $\eta:[-h, h] \rightarrow U$ be the straight line between $x$ and $z$ parametrised proportional to the Euclidean arc length and put $m=\eta(0)=\frac{1}{2}(x+z)$. We will estimate the distance $|y-m|_{x}$.

Denote the geodesic sub-segment of $\gamma$ connecting $x$ and $y$ by $\gamma_{1}$. By Corollary 3.3 we have $|x-y|_{x} \leq L_{x}\left(\gamma_{1}\right) \leq L\left(\gamma_{1}\right)+C L\left(\gamma_{1}\right)^{1+\alpha} \leq h+C h^{1+\alpha}$. Similarly denote $\gamma([0, h])$ by $\gamma_{2}$. Thus $|y-z|_{x} \leq|y-z|_{y}+C|| y-z|| \| x-y||^{\alpha} \leq L\left(\gamma_{2}\right)+C L\left(\gamma_{2}\right)^{1+\alpha}+$ $C^{2} d(y, z)(C d(x, y))^{\alpha} \leq h+\left(C+C^{3}\right) h^{1+\alpha}$. Moreover, we have $|x-z|_{x} \geq d(x, z)-$ $C d(x, z)^{1+\alpha} \geq 2 h-4 C h^{1+\alpha}$.

Finally, since $|\cdot|$ is $\kappa$-convex of type $p$, by applying Lemma 2.3 for $K=$ $\max \left\{C+C^{3}, 4 C\right\}$ we see that $|y-m|_{x} \leq 2 \lambda h^{1+\frac{\alpha}{p}}$ for all $h \leq \epsilon$, where $\lambda=\lambda(K, \kappa)$ and $\epsilon=\epsilon(K, \kappa)$ are the constants given in Lemma 2.3. This means exactly that $\|\gamma(-h)+\gamma(h)-2 \gamma(0)\| \leq 2 \lambda h^{1+\frac{\alpha}{p}}$, and hence by Lemma 2.2 this implies Theorem 1.3 .

4.2. Better estimations and the proof of Theorem 1.4. Before we give the proof of Theorem 1.4 we make two trivial remarks that are used in the following arguments.

Remark 4.1. Let $f:[a, b] \rightarrow \mathbb{R}$ be a positive continuous function. Then

$$
\|b-a\| \int_{a}^{b} f^{2}(t) d t \geq\left(\int_{a}^{b} f(t) d t\right)^{2} .
$$

Remark 4.2. There exists an $\epsilon$ (for example $\epsilon=\frac{1}{10}$ ) such that for all real numbers $x, a, b>0$ with $\|1-x\|+\|a\|+\|b\|<\epsilon$ one has $\sqrt{x+a-b} \geq \sqrt{x}+\frac{1}{3} a-b$. In fact this follows directly from Taylor expansion of the square root function.

Let $U$ be a chart with the properties as defined in the beginning of the section, and assume in addition, that all tangent spaces $\left(T_{x} U,|\cdot|{ }_{x}\right)$ are Euclidean spaces, i.e. the norm $|\cdot|_{x}$ stems from the a scalar product $\langle\cdot, \cdot\rangle_{x}$. Therefore the norm is $\kappa$-convex of type 2 with $\kappa \geq \frac{1}{10}$ (see Example 2.2).

Again let $h$ be a small number, $\gamma:[-h, h] \rightarrow U$ a geodesic, $x=\gamma(-h), z=\gamma(h)$, $y=\gamma(0)$ and $m=\frac{1}{2}(x+z)$. We may assume that $m$ is the origin of $\mathbb{R}^{n}$ and that the norm $|\cdot|_{0}$ is the Euclidean norm $\|\cdot\|$. Set $e=\frac{z}{\|z\|}$ and denote by $P$ the line generated by $e$. Let $H$ be the orthogonal hyperplane to $P$. Remark that for 
each unit vector $h \in H$ and all $a \in U$ we have $\langle e, h\rangle_{a}=\frac{1}{4}\left(|e+h|_{a}^{2}-|e-h|_{a}^{2}\right)=$ $\left(|e+h|_{a}^{2}-|e+h|_{0}^{2}\right)+\left(|e-h|_{0}^{2}-|e-h|_{a}^{2}\right)$. Since $\|\left. v\right|_{x}-|v|_{y}|\leq C||x-y|^{\alpha+1}$ for all $x, y$ and $v$ with $\|v\|=1$, we obtain $\langle e, h\rangle_{a} \leq 10 C^{2}(\|e+h\|+\|e-h\|)\|a\|^{\alpha} \leq 10 C^{2}\|a\|^{\alpha}$.

We decompose $\gamma(t)$ as $\gamma(t)=f(t) e+v(t)$, with $v(t) \in H$ and $f(t) \in \mathbb{R}$. By Theorem 1.3 the geodesics are uniformly locally $\mathcal{C}^{1, \frac{\alpha}{2}}$. Therefore $f$ and $v$ are of class $\mathcal{C}^{1}$ and by Lemma 2.1 choosing $h$ small enough we may assume $\left\|v^{\prime}(t)\right\| \leq \epsilon$ for arbitrary small $\epsilon>0$. Since $\left|\gamma^{\prime}(t)\right|_{\gamma(t)}=1$ we see that $f^{\prime}(t)|e|_{\gamma(t)} \geq 1-\epsilon$.

Consider the straight line $\eta:[-h, h] \rightarrow U$ between $x$ and $z$ parametrised by $\eta(t)=f(t) e$. We see that $\gamma(t)=\eta(t)+v(t)$. Moreover, $1-f^{\prime}(t)|e|_{\gamma(t)} \leq \epsilon$ and $\left\|v^{\prime}(t)\right\| \leq \epsilon$ for arbitrary $\epsilon$, and we can use Remark 4.2 to obtain

$$
\begin{aligned}
1 & =\left|\gamma^{\prime}(t)\right|_{\gamma(t)}=\left(f^{\prime}(t)^{2}|e|_{\gamma(t)}^{2}+\left|v^{\prime}(t)\right|_{\gamma(t)}^{2}+2 f^{\prime}(t)\left\langle e, v^{\prime}(t)\right\rangle_{\gamma(t)}\right)^{\frac{1}{2}} \\
& \geq f^{\prime}(t)|e|_{\gamma(t)}+\frac{1}{3}\left(\left|v^{\prime}(t)\right|_{\gamma(t)}^{2}\right)-2\left|f^{\prime}(t)\left\langle e, v^{\prime}(t)\right\rangle_{\gamma(t)}\right| \\
& \geq f^{\prime}(t)|e|_{\eta(t)}-C|| v(t)\left\|^{\alpha}+\frac{1}{C^{\prime}}\right\| v^{\prime}(t)\left\|^{2}-C^{\prime}|| v^{\prime}(t)\right\|(\|\gamma(t)\|)^{\alpha},
\end{aligned}
$$

where $C^{\prime}=\max \left\{\frac{C^{2}}{3}, 40 C\right\}$.

Moreover $L(\gamma) \leq L(\eta)=\int_{-h}^{h} f^{\prime}(t)|e|_{\eta(t)} d t$, since $\gamma$ is a geodesic. This, together with the above inequality, implies

$$
\frac{1}{C^{\prime}} \int_{-h}^{h}\left\|v^{\prime}(t)\right\|^{2} \leq C^{\prime} \int_{-h}^{h}\left(\|v(t)\|^{\alpha}+\left\|v^{\prime}(t)\right\|\|\gamma(t)\|^{\alpha}\right) d t
$$

Using $\|\gamma(t)\| \leq\|v(t)\|+2\|t\|$ and $\left\|v^{\prime}(t)\right\| \leq 1$, we see that $\int_{-h}^{h}\left\|v^{\prime}(t)\right\|^{2} \leq$ $\frac{1}{2} K\left(\int_{-h}^{h}(\|v(t)\|)^{\alpha}+\left\|v^{\prime}(t)\right\|\|t\|^{\alpha} d t\right)$, where $K=2 C^{\prime 2}$. Thus one has either

$$
\int_{-h}^{h}\left\|v^{\prime}(t)\right\|^{2} d t \leq K \int_{-h}^{h}\left(\|v(t)\|^{\alpha}\right) d t
$$

or

$$
\int_{-h}^{h}\left\|v^{\prime}(t)\right\|^{2} d t \leq K \int_{-h}^{h}\left\|v^{\prime}(t)\right\|\|t\|^{\alpha} d t
$$

Consider the maximum $v_{0}=\max _{-h \leq t \leq h}\|v(t)\|$. Since $v(h)=v(-h)=0$, we have $v_{0} \leq \int_{-h}^{h}\left\|v^{\prime}(t)\right\| d t$. In case (2) we get from the Cauchy Schwartz inequality that $\int_{-h}^{h}\left\|v^{\prime}(t)\right\|^{2} d t \leq K\left(\int_{-h}^{h}\left\|v^{\prime}(t)\right\|^{2} d t\right)^{\frac{1}{2}}\left(\int_{-h}^{h}\|t\|^{2 \alpha} d t\right)^{\frac{1}{2}}$. This implies $\int_{-h}^{h}\left\|v^{\prime}(t)\right\|^{2} d t \leq 2 K^{2} h^{2 \alpha+1}$, and using Remark 4.1 we obtain $v_{0} \leq 2 K^{2} h^{\alpha+1}$. In case (1), we obtain $\left(\int_{-h}^{h}\left\|v^{\prime}(t)\right\| d t\right)^{2} \leq 2 K h \int_{-h}^{h}\left(\|v(t)\|^{\alpha}\right) d t \leq 4 K h^{2} v_{0}^{\alpha}$ using Remark 4.1. Therefore $v_{0}^{2} \leq 4 K h^{2} v_{0}^{\alpha}$. That implies $v_{0} \leq K^{\prime} h^{\frac{2}{2-\alpha}}$, where $K^{\prime}=(4 K)^{\frac{1}{2-\alpha}}$.

Since for $\beta=\frac{\alpha}{2-\alpha}$ we have $\beta<\alpha$ and $\frac{2}{2-\alpha}=1+\beta$, for each $t \in[-h, h]$ we obtain: $\|\gamma(t)-\eta(t)\|=C\|v(t)\| \leq C v_{0} \leq A h^{1+\beta}$, for some $A$ depending only on $C$.

In order to apply Lemma 2.2 we have to show that $\|\gamma(0)-m\| \leq L h^{1+\beta}$ for some constant $L$ depending only on $C$. Since $m$ is the origin we have $\| \gamma(0)-$ $m\|\leq\| \gamma(0)-\eta(0)\|+\| \eta(0)\left\|\leq A h^{1+\beta}+\right\| f(0) e \|$. Thus we only have to show $\|f(0) e\| \leq L h^{1+\beta}$ for some fixed $L$. 
Assume $\|f(0) e\| \geq L h^{1+\beta}$ with $L \geq 2 C^{2}$. We may assume that $f(0)<0$. We have $|x|_{x}=|x-m|_{x} \leq h+C h^{1+\alpha}$. Thus $|x-\eta(0)|_{x} \leq h+C h^{1+\alpha}-|f(0) e|_{x} \leq$ $h-\frac{L}{2 C} h^{1+\beta}$. Then $|x-\gamma(0)|_{x} \leq|x-\eta(0)|_{x}+|\eta(0)-\gamma(0)|_{x} \leq h-\frac{L}{2 C} h^{1+\beta}+A C h^{1+\beta}$. If $L \geq 4 A C^{2}$ we get $|x-\eta(0)|_{x} \leq h-\frac{L}{4 C} h^{1+\beta}$. Using Corollary 3.4 we get a contradiction to $d(x, \gamma(0))=h$. Thus we have proved $L \leq 4 A C^{2}$ and the proof of Theorem 1.4 is finished.

\section{The COUnterexample}

Let $0<\alpha<1$ be fixed and set $\beta=\frac{\alpha}{2-\alpha}$. Consider in $\mathbb{R}^{2}$ the graph $\Gamma$ of the function $f: \mathbb{R} \rightarrow \mathbb{R}$ given by $f(t)=|t|^{1+\beta}$. Set $\rho(x)=d(x, \Gamma)=\min _{y \in \Gamma}\{\| x-y||\}$. Denote by $\tilde{g}$ the Euclidean metric and consider on $\mathbb{R}^{2}$ the metric $g_{x}=\left(1+A \rho(x)^{\alpha}\right) \tilde{g}$, where $A$ is a constant $\geq 100$. This metric is $\alpha$-Hölder, and it is locally Lipschitz outside of $\Gamma$. At each point of $\Gamma$ the new metric $g$ coincides with the Euclidean metric $\tilde{g}$. For $t \in \mathbb{R}$ denote by $x_{t}$ the point $(t, f(t))$ on $\Gamma$. We will prove that the geodesics between $x_{-t}$ and $x_{t}$ cannot be uniformly $\mathcal{C}^{1, l}$ for any $l>\beta$, i.e. we show that geodesics between $x_{-t}$ and $x_{t}$ must be very close to $\Gamma$.

The length of the curve $\gamma_{t}:[-t, t] \rightarrow \mathbb{R}^{2}$ defined by $\gamma_{t}(s)=(s, f(s))=x_{s}$ is given by $L\left(\gamma_{t}\right)=L_{e}\left(\gamma_{t}\right)=\int_{-t}^{t} \sqrt{1+(1+\beta)^{2} s^{2 \beta}} d s$. Hence by Remark 4.2 and the fact that $\beta \leq 1$, we have for all sufficiently small $t, L\left(\gamma_{t}\right) \leq \int_{-t}^{t}\left(1+2 s^{2 \beta}\right) d s \leq$ $2 t+10 t^{2 \beta+1}$.

Now fix a small relatively compact neighbourhood $U$ of 0 and assume that geodesics in $U$ are uniformly $\mathcal{C}^{1, l}$ for some $l>\beta$. Let $\eta_{t}:[-h, h] \rightarrow \mathbb{R}^{2}$ be a geodesic between $x_{-t}$ and $x_{t}$. Hence for all $s, r$ in $[-t, t]$ we have $\left\|\eta_{t}^{\prime}(s)-\eta_{t}^{\prime}(r)\right\| \leq C\|s-r\|^{l}$, where $C$ is a constant depending only on $U$. By Lemma 2.1 we see that for each $r \in[-h, h]$ the distance between $\eta_{t}(r)$ and the affine line between $x_{-t}$ and $x_{t}$ is at most $C t^{1+l}$.

We reparametrise $\eta_{t}$ by the first coordinate and denote the new curve by $\tilde{\eta}_{t}$ : $[-t, t] \rightarrow \mathbb{R}^{2}$, where $\tilde{\eta}_{t}(s)=\left(s, y_{t}(s)\right)$. From above we know $y_{t}(s) \geq f(t)-C t^{l+1}=$ $t^{\beta+1}\left(1-C t^{l-\beta}\right)$. Since $l>\beta$ for arbitrary $\epsilon>0$ and all $t$ small enough, we get $y_{t}(s) \geq f(t)(1-\epsilon) \geq \frac{2}{3} f(t)$.

For each $x_{r}=(r, f(r)) \in \Gamma$ one has either $|r| \geq \frac{t}{2}$ or $f(r) \leq \frac{t^{1+\beta}}{2}$. Hence for each $s$ with $|s| \leq \frac{t}{3}$ we get $d\left(\tilde{\eta}_{t}(s), \Gamma\right) \geq \frac{1}{6} t^{1+\beta}$.

Now we can estimate the length of $\eta_{t}$ by

$$
\begin{aligned}
L\left(\eta_{t}\right) & =L\left(\tilde{\eta}_{t}\right) \geq L_{\epsilon}\left(\tilde{\eta}_{t}\right)+\int_{-t}^{t} A\left(d\left(\tilde{\eta}_{t}(r), \Gamma\right)\right)^{\alpha} d r \\
& \geq 2 t+A \int_{-\frac{t}{3}}^{\frac{t}{3}}\left(\frac{1}{6} t^{1+\beta}\right)^{\alpha} d r=2 t+\frac{100}{9} t^{1+\alpha+\alpha \beta} .
\end{aligned}
$$

But by our assumption $\eta_{t}$ is a geodesic, hence $L\left(\eta_{t}\right) \leq L\left(\gamma_{t}\right)$. We get $10 t^{2 \beta+1} \geq$ $\frac{100}{9} t^{1+\alpha+\alpha \beta}$. Since $1+\alpha+\alpha \beta=1+2 \beta$, this is a contradiction.

\section{REFERENCES}

[CH70] E. Calabi and Ph. Hartmann. On the smoothness of isometries. Duke Math. J., 37:741750, 1970. MR0283727(44:957)

[Kos02a] N. N. Kosovskii. On gluing of Riemannian manifolds of curvature $\geq \kappa$. Algebra i Analiz, 14:140-157, 2002. MR1921991 (2003d:53053) 
[Kos02b] N. N. Kosovskii. On gluing of Riemannian manifolds of curvature $\leq \kappa$. Algebra $i$ Analiz, 14:73-86, 2002. MR 1970333 (2004g:53044)

[LT79] J. Lindenstrauss and L. Tzafriri. Classical Banach spaces.Function spaces, volume 2. Springer-Verlag, 1979. MR0540367 (81c:46001)

[Lyt] A. Lytchak. Almost convex subsets. Preprint.

[Tay] M. Taylor. Existence and regularity of Isometries. Preprint.

Department of Mathematics, University of Bonn, 53115 Bonn, Germany

Department of Mathematics, University of Bonn, 53115 Bonn, Germany

Current address: CRM, Apartat 50, E-08193, Bellaterra, Spain 